\title{
Mutations in the netrin-1 gene cause congenital mirror movements
}

\author{
Aurélie Méneret, ${ }^{1,2}$ Elizabeth A. Franz, ${ }^{3}$ Oriane Trouillard, ${ }^{1}$ Thomas C. Oliver, ${ }^{4}$ Yvrick Zagar, ${ }^{5}$ Stephen P. Robertson, ${ }^{6}$ \\ Quentin Welniarz, ${ }^{1,7}$ R.J. MacKinlay Gardner, ${ }^{6}$ Cécile Gallea, ${ }^{1}$ Myriam Srour, ${ }^{8,9}$ Christel Depienne, ${ }^{1,10,11}$ Christine L. Jasoni, ${ }^{12}$ \\ Caroline Dubacq, ${ }^{7}$ Florence Riant, ${ }^{13,14}$ Jean-Charles Lamy, ${ }^{1}$ Marie-Pierre Morel, ${ }^{7}$ Raphael Guérois, ${ }^{15}$ Jessica Andreani, ${ }^{15}$ \\ Coralie Fouquet, ${ }^{7}$ Mohamed Doulazmi, ${ }^{16}$ Marie Vidailhet, ${ }^{1,2}$ Guy A. Rouleau, ${ }^{8,1718}$ Alexis Brice, ${ }^{1,19}$ Alain Chédotal, ${ }^{5}$ \\ Isabelle Dusart, ${ }^{7}$ Emmanuel Roze, ${ }^{1,2}$ and David Markie ${ }^{4}$
}

IINSERM U1127, CNRS UMR 7225, Sorbonne Universités, UPMC Université Paris 06, UMR S1127, CIC-1422, Institut du Cerveau et de la Moelle épinière (ICM), Paris, France. 'AP-HP, Hôpital de la Pitié-Salpêtrière, Département de Neurologie, Paris, France. ${ }^{3}$ Department of Psychology and fMRlotago, University of Otago, Dunedin, New Zealand. PPathology Department, Dunedin School of Medicine, University of Otago, Dunedin, New Zealand. 5Sorbonne Universités, UPMC Université Paris 06, INSERM, CNRS, Institut de la Vision, Paris, France. 'Department of Women's and Children's Health, Dunedin School of Medicine, University of Otago, Dunedin, New Zealand. 'S Sorbonne Universités, UPMC Université Paris 06, INSERM, CNRS, Institut de Biologie Paris Seine, Neuroscience Paris Seine, Paris, France. ${ }^{8}$ Department of Neurology and Neurosurgery, and ${ }^{9}$ Department of Paediatrics, McGill University, Montreal, Quebec, Canada. ${ }^{10}$ Institut de Cénétique et de Biologie moléculaire et cellulaire (ICBMC), CNRS UMR 7104, INSERM U964,

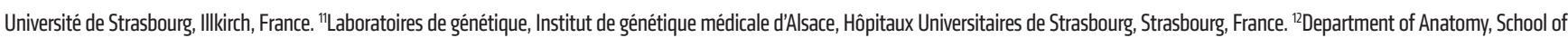
Biomedical Sciences, University of Otago, Dunedin, New Zealand. ${ }^{13}$ AP-HP, Groupe hospitalier Lariboisière-Fernand Widal, Laboratoire de Cénétique, Paris, France. ${ }^{14}$ INSERM, UMR S740, Université Paris 7 Denis Diderot, Paris, France. ${ }^{15}$ nstitute for Integrative Biology of the Cell (I2BC), CEA, CNRS, Université Paris Sud, Université Paris-Saclay, Gif sur Yvette, France. ${ }^{16}$ Sorbonne Universités, UPMC Université Paris 06, INSERM, CNRS, Institut de Biologie Paris Seine, Adaptation Biologique et Vieillissement, Paris, France. ${ }^{17}$ Montreal Neurological Institute, Montreal, Quebec, Canada. ${ }^{18}$ Department of Human Cenetics, McGill University, Montreal, Quebec, Canada. ${ }^{19} \mathrm{AP}$-HP, Hôpital de la Pitié-Salpêtrière, Fédération de Cénétique, Département de Cénétique et de Cytogénétique, Paris, France.

\begin{abstract}
Netrin-1 is a secreted protein that was first identified 20 years ago as an axon guidance molecule that regulates midline crossing in the CNS. It plays critical roles in various tissues throughout development and is implicated in tumorigenesis and inflammation in adulthood. Despite extensive studies, no inherited human disease has been directly associated with mutations in NTN1, the gene coding for netrin-1. Here, we have identified 3 mutations in exon 7 of NTN1 in 2 unrelated families and 1 sporadic case with isolated congenital mirror movements (CMM), a disorder characterized by involuntary movements of one hand that mirror intentional movements of the opposite hand. Given the diverse roles of netrin-1, the absence of manifestations other than CMM in NTN1 mutation carriers was unexpected. Using multimodal approaches, we discovered that the anatomy of the corticospinal tract (CST) is abnormal in patients with NTN1-mutant CMM. When expressed in HEK 293 or stable HeLa cells, the 3 mutated netrin-1 proteins were almost exclusively detected in the intracellular compartment, contrary to WT netrin-1, which is detected in both intracellular and extracellular compartments. Since netrin-1 is a diffusible extracellular cue, the pathophysiology likely involves its loss of function and subsequent disruption of axon guidance, resulting in abnormal decussation of the CST.
\end{abstract}

\section{Introduction}

Netrins are a family of extracellular proteins that regulate cell migration and survival during development and adulthood. The first member, netrin-1, was identified as a secreted protein mediating axon guidance in the spinal cord (1). Netrin-1 is expressed at the midline of the developing CNS and acts as an attractive or repulsive cue for different populations of crossing/commissural axons (2). The classical view that attraction of commissural axons is mediated by a gradient of floor-plate-derived netrin- 1 has been recently challenged: netrin-1 primarily acts locally by promoting growth cone adhesion $(3,4)$. In netrin-1-KO mice, many commissural axons fail to cross the midline in the corpus callosum, the hip-

Authorship note: E. Roze and D. Markie contributed equally to this work. Conflict of interest: The authors have declared that no conflict of interest exists. Submitted: June 5, 2017; Accepted: August 2, 2017.

Reference information: J Clin Invest. 2017;127(11):3923-3936.

https://doi.org/10.1172/JCI95442. pocampal and anterior commissures, the corticospinal tract (CST), and the spinal cord (5-7). Netrin-1 binds to several transmembrane receptors including deleted in colorectal carcinoma (DCC) (8) and UNC5s, which respectively mediate attractive or repulsive axonal responses to netrin-1.

Netrin-1 exerts a variety of functions beyond axon guidance and is involved in synaptogenesis, apoptosis, cell migration, and angiogenesis $(9,10)$. There is also evidence suggesting that netrin-1 influences remyelination and tumorigenesis $(11,12)$. Netrin-1 is a potential therapeutic target and/or biomarker in cancer, cardiovascular disease, neuroinflammation, and kidney disease (12-14). However, except for a possible association with cleft lip in GWAS studies (15), the 20th anniversary of the discovery of netrin-1 has passed without the identification of a single human disease caused by inherited mutations in NTN1, the gene coding for netrin-1. In contrast, variants in the genes coding for receptors of netrin-1 such as UNC5C have been linked to Alzheimer's disease and colorectal cancer (16), while mutations in DCC have 
A

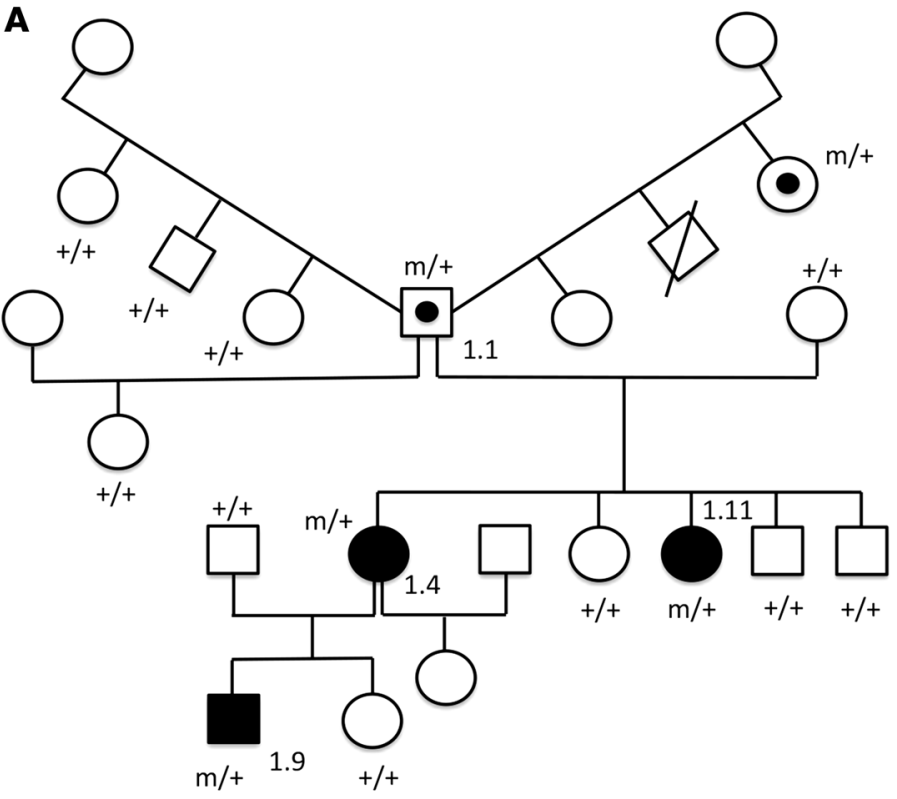

B

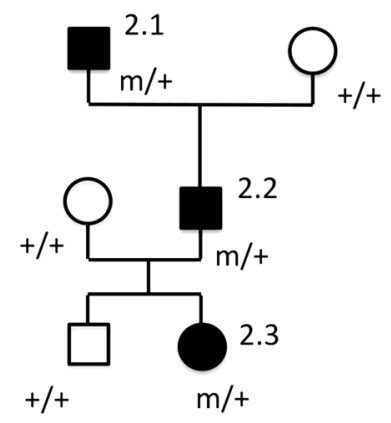

C G T T A A C A CATCT C C T G T

Control

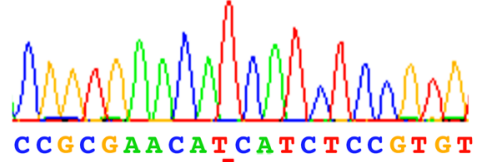

Patient

2.3

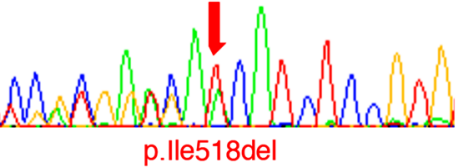

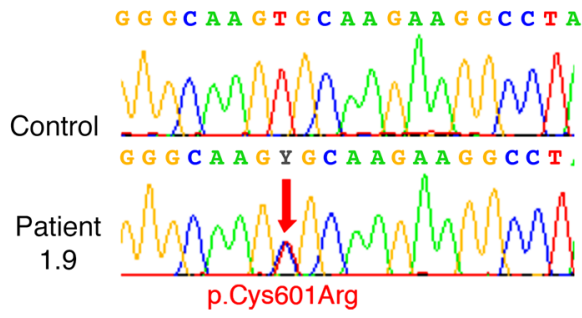

C

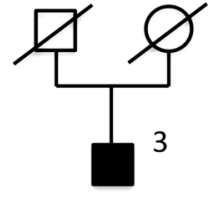

$\mathrm{m} /+$

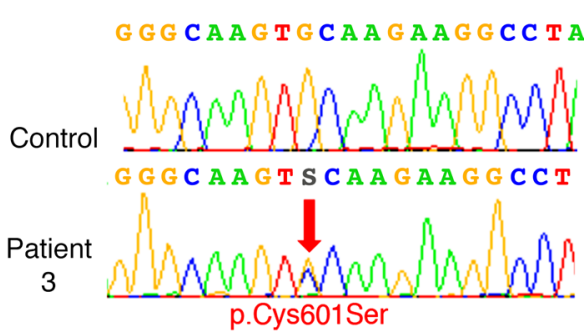

Figure 1. Pedigrees of the CMM families and distribution of the mutations in NTN1. (A) Family 1 (from France) with a C601R mutation (the unusual family tree is a result of individual 1.1, who had children with 4 different women), (B) family 2 (from the United Kingdom [data were collected in New Zealand], which was previously identified as Family C in ref. 33), with an I518del mutation, and (C) a Canadian sporadic case with a C601S mutation. Black symbols represent individuals with CMM, symbols with an embedded black circle indicate asymptomatic carriers, white symbols indicate unaffected individuals, and symbols with a diagonal line represent deceased individuals. Squares represent males and circles represent females. m, mutated allele; +, WT allele. Electrophoregrams confirm the 3 NTN1 mutations obtained by Sanger sequencing. Red arrows point to sequence changes. Electrophoregrams were obtained with SeqScape software, version 2.6 (Applied Biosystems).

been shown to cause agenesis of the corpus callosum (17), splitbrain syndrome (18), and the rare disorder of congenital mirror movements (CMM) (19-21).

CMM is characterized by involuntary movements of one side of the body that accompany and mirror intentional movements on the opposite side (Online Mendelian Inheritance in Man [OMIM] \#157600) $(22,23)$. CMM mostly involves the hands. Affected subjects are unable to perform pure unimanual movements and have difficulty with skills requiring dissociated movements of the 2 hands $(24,25)$. It has been described as a familial disorder with autosomal dominant inheritance and incomplete penetrance, but sporadic cases also exist. The pathophysiology of CMM is probably related to developmental abnormalities of the CST, which conveys motor outputs from the motor cortex to the contralateral side of the body, and of the corpus callosum, which underlies communication between motor cortices. CMM usually involves abnormal decussation of the CSTs and altered interhemispheric inhibition with bilateral activation of the primary motor cortices during unimanual movements (26-28). To date, the established culprit genes are $D C C$ and RAD51, which encodes a DNA repair protein, and possibly DNAL4, which encodes an axonemal dynein light chain (19, 21, 29-31). Recently, RAD51 has been described as a negative regulator of the netrin-1 signaling pathway (32). Only $35 \%$ of affected individuals or families carry a pathogenic variant in 1 of these 3 genes (22). This study has been undertaken to investigate whether mutations in other axon guidance genes, particularly those interacting with DCC or belonging to the netrin-1/DCC pathway, might cause CMM.

\section{Results}

Three netrin-1 variants are associated with CMM. Exome sequencing was conducted in 25 index cases of CMM (8 familial and 17 sporadic) without mutations in DCC, RAD51, or DNAL4. Patients' characteristics and genetic findings are summarized in Supplemental Table 1; supplemental material available online with this article; https://doi.org/10.1172/JCI95442DS1. More 
than 35,000 variants were identified per individual (Supplemental Tables 2 and 3). The flow chart of exome data analysis is summarized in Supplemental Figure 1. We initially filtered the data to remove variants that are too common to explain the low prevalence of the disorder (allele frequency greater than 0.1\% in the Exome Variant Server [EVS] and 1000 Genomes databases; see Methods), those unlikely to affect protein-coding genes (intronic and synonymous), and low-confidence variant calls owing to poor coverage in our data (read depths of less than $10 \times$ in the French exomes and less than $5 \times$ in the New Zealand exomes) (Supplemental Table 4). Genes were then prioritized as candidates by known connection to $D C C$ and RAD 51 function by the number of unrelated individuals with variants, the functional prediction scores, and the rarity of variants in the Exome Aggregation Consortium (ExAC) database (comprising exome data on 60,706 unrelated individuals; see Methods). A total of 17 genes, with variants in at least 3 index cases or in 2 index cases but with a known function in axon guidance or known interaction with $D C C$ or RAD51, were selected (Supplemental Figure 1). Interestingly, the NTN1 gene, coding for netrin-1, was among them, with 2 individuals carrying heterozygous variants. One missense variant (c.1801T >C/p.Cys601Arg) of NTN1 segregated with CMM in all three affected members of a French family (Figure 1A) was predicted to be pathogenic by Sorting Intolerant From Tolerant (SIFT), MutationTaster, and PolyPhen-2 algorithms and was absent from the ExAC database (Table 1). An in-frame deletion (c.1552_1554del/p.Ile518del) of NTN1 segregated with CMM in all 3 affected members of a family from the United Kingdom (data collected in New Zealand; Family C in ref. 33) (Figure 1B) and was also predicted to be pathogenic (Table 1). To confirm the role of NTN1 in CMM, we sequenced the NTN1 gene in 22 additional CMM index cases (20 sporadic and 2 familial cases from a Canadian cohort, all negative for DCC, RAD51, and DNAL4) and found another missense variant of NTN1 (c.1802G>C/ p.Cys601Ser) in a sporadic case (Figure 1C). This variant was also predicted to be pathogenic (Table 1). These 3 variants alter amino acids in the NTR (netrin) domain (Figure 2A). ExAC data showed a rare variant in Ile518 (Ile518Val, 2 of 119,708 alleles), but valine has physicochemical properties similar to those of isoleucine. No variant affecting the 6 conserved cysteines (C472, C476, C491, C544, C546, and C601) of the NTR domain was present in ExAC, except for a synonymous variant in C472. ExAC data showed several rare $N T N 1$ variants (allele frequency $<0.1 \%$ ) predicted to be pathogenic by both SIFT and PolyPhen-2 (Supplemental Table 5) and 2 rare loss-of-function variants, which have not been confirmed. Statistical analysis showed that the frequency of NTN1 variants predicted to be deleterious was significantly different between patients with CMM and the ExAC controls (3 of 47 vs. 63 of 60,$706 ; P=2.306 \times 10^{-5}$ ). In addition, all 3 variants described here in CMM patients (I518del, C601R, C601S) alter amino acids that are highly conserved among vertebrates (Figure 2C). Because the precise structure of the NTR domain of netrin-1 has not yet been determined experimentally through crystallography, we predicted its structure by using Rosetta comparative modeling software (34). This structural model of the NTR domain revealed that Cys601 is probably involved in a conserved disulfide bridge with Cys491 found in many members of

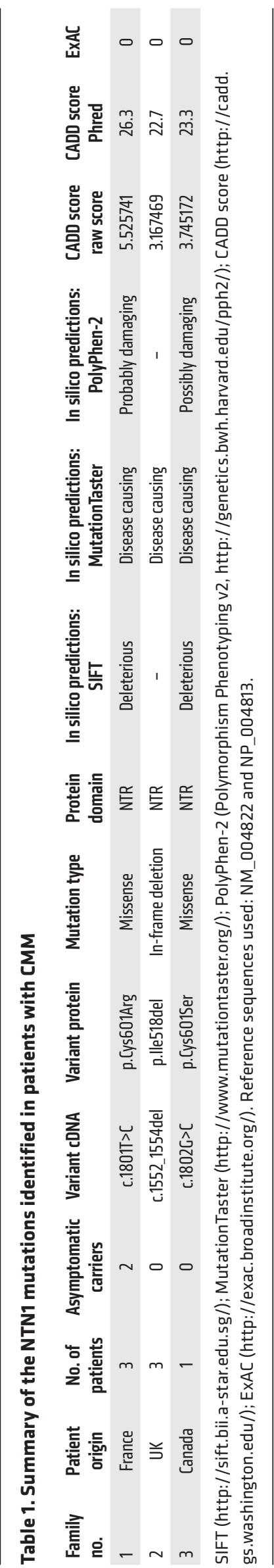


A

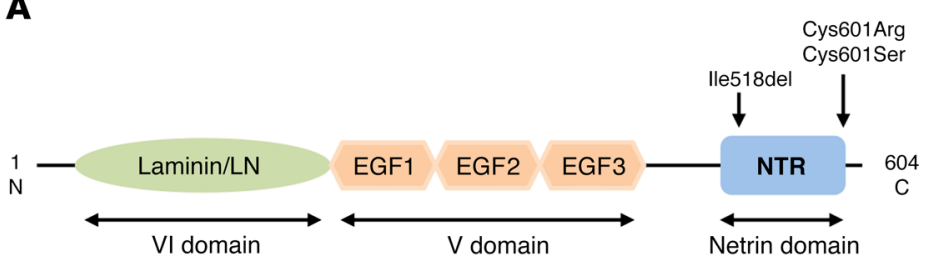

B

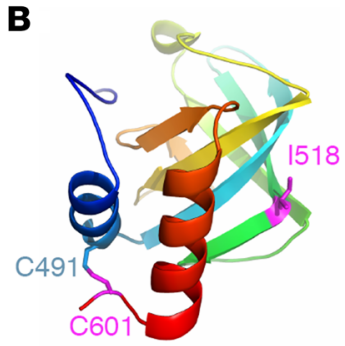

C

Netrin-1 orthologs

NTN1_Human
NTN1_Macaque
NTN1_Vervet-monkey
NTN1_Mouse
NTN1_Rat
NTN1_Cow
NTN1_Dog
NTN1_Chicken
NTN1_Anole-1izard
NTN1_Zebrafish
NTN1_Tetraodon

NTN1 Human NTN1_Macaque NTN1_Vervet-monkey NTN1_Mouse NTN1 Rat NTN1 Cow NTN1_Dog NTN1 Chicken NTN1_Anole-lizard NTN1 Zebrafish NTN1_Tetraodon

518

NMKKYCKKDYAVQIHILKADKAGDWWKFTVNIISVYKQGTSRIRRGD NMKKYCKKDYAVQIHILKADKAGDWWKFTVNIISVYKQGTSRIRRGD NMKKYCKKDYAVQIHILKADKAGDWWKFTVNIISVYKQGTSRIRRGD NMKKYCRKDYAVQIH ILKADKAGDWWKFTVNI ISVYKOGTSRIRRGD NMKKYCRKDYAVQIHILKADKAGDWWKFTVNI ISVYKQGTSRIRRGD NMKKYCKKDYAVOIHILKADKAGDWWKFTVNIISVYKOGTSRIRRGD MKKYCRK-DYAVQIHILKADKAEDWWKFTVNIIS-YKOGTSRIRRGD NMKKYCKKDYAVQIHI-KAEKNADWWKFTVNI ISVYKQGSNRIRRGD NMKKYCKKDYAVQIH ILKADKAGEWWKFTVNI I SVYKQGTNRIRRGD TMKKYCKKDFAVQVHVLKGDKAGEWWKFTINI ISVYKQGGHRIRRGD NMKKYCKKDYAVOIHILKADKAGEWWKFTVNI ISVYKOGESRIRRGD $. * * * * *: * *: * * *: *: * * .: * \quad: * * * * *: * * * * * * * * \quad *: * * * *$

601

SSLVIQWRDTWARRLRKFQQREKKGKCKKA SSLVIQWRDTWARRLRKFQQREKKGKCKKA SSLVIQWRDTWARRLRKFQQREKKGKCKKA SSLVIQWRDTWARRLRKFQQREKKGKCKKA SSLVIQWRDTWARRLRKFQQREKKGKCKKA SSLVIQWRDTWARRLRKFQQREKKGKCKKA SSLVIQWRDTWARRLRKFQQREKKGKCKKA SSLVIQWRDTWARRLRKFQQREKKGKCRKA SSLVIQWRDTWARRLRKFQQREKKGKCKKA GSLLIPWKDLWARRLRKFOORDKRGKC--GSLVIQWRDTWARRLRKFQQREKKGKCKKP

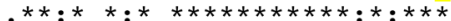

Figure 2. Position and conservation of the mutated amino acids. (A) Schematic of the netrin-1 protein showing the laminin (LN) domain, the 3 EGF-like domains, and the netrin (NTR) domain. Arrows show the location of the 3 mutations. (B) Structural model of the NTR domain of WT netrin-1 showing the 2 cysteines involved in a disulfide bridge (C601 and C491) and I518, which has a key position in a $\beta$-strand. The 2 mutated amino acids (I518 and C601) are in purple. (C) Alignment of the regions flanking the 3 variants in vertebrate netrin-1 orthologous proteins, showing the conservation of the altered amino acids. Multiple pairwise alignments were performed using Clustal Omega (http://www.ebi. ac.uk/Tools/msa/clustalo/). The amino acids altered by the mutations are highlighted. Asterisk indicates a fully conserved residue; colon indicates conservation between groups of strongly similar properties; period indicates conservation between groups of weakly similar properties. the superfamily of NTR domains and that Ile518 has a key position in a $\beta$-strand (Figure 2B) (35-37). Altogether, 3 NTN1 variants from 3 independent families were associated with CMM and predicted to be pathogenic by the SIFT, MutationTaster, and PolyPhen-2 algorithms, the combined annotation-dependent depletion (CADD) score, and by the structural model.

Patients harboring NTN1 variants have abnormal crossing of the CST. Clinically, the NTN1 patients (including an 81-yearold asymptomatic carrier) had normal eyesight, no oculomotor abnormalities, no cardiovascular or respiratory disease, no inflammatory disease, and no cancer. Two of the patients had childhood-onset constipation-dominant irritable bowel syndrome (IBS), and two had peripheral vasoconstriction when exposed to cold (Supplemental Table 1). Except for mirror movements, neurological examination was normal. MRI analysis was performed for 1 patient (patient 1.9) and 20 control subjects. A brain MRI of this patient was normal, showing no corpus callosum hypoplasia or visible pontine abnormalities (Supplemental Figure 2). Tractography can provide individualized volumes of interest for the investigation of white matter microstructural characteristics in the context of disease. Tracts are personalized to each individual, and their positions are subject to interindividual anatomical variability. The variation of fractional anisotropy (FA) (the basic diffusion tensor imaging [DTI] metric) throughout the white matter is informative, for instance, with low values at the transition between gray and white matter or at the crossing of 2 main pathways. Therefore, we performed tract-based analyses that allowed between-subjects comparisons and optimized the localization of within-tract alterations (Figure 3, A and B). First, for each subject, we calculated the CST laterality coefficient on the basis of ratio between the number of streamlines crossing the midline and the number of streamlines projecting to the ipsilateral side after the pyramidal decussation. Comparison of the CST laterality coefficient of patient 1.9 with that of a group of 20 controls using the Crawford-Howell $t$ test showed a significant group difference ( $P=0.001$; Figure $3 C)$ (34). Second, we performed between-subjects analysis of FA values along the length of the crossed CST, focusing on the pyramidal decussation. We investigated the projection of the CST in 1 patient and 1 control subject (age and sex matched, Figure 3D). Mean FA values increased along the crossed CST in the patient compared with the control subject at the level of the pyramidal decussation (Figure 3D). The relative increased FA value at this location would suggest that fewer axons are crossing the midline in the patient. These results suggested that a higher proportion of fibers was located in the crossed versus the uncrossed CST in the control subject, whereas a higher proportion of fibers was located in the uncrossed CST in the patient (Figure 3D). We then used single-pulse transcranial 
A

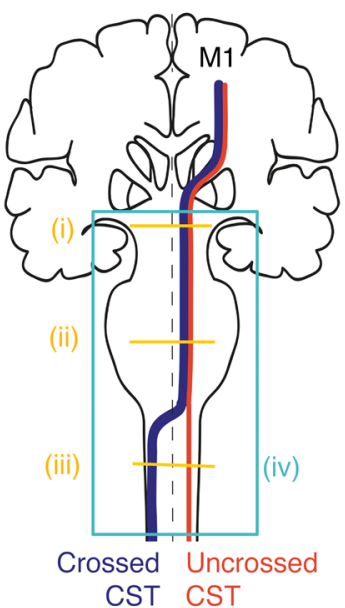

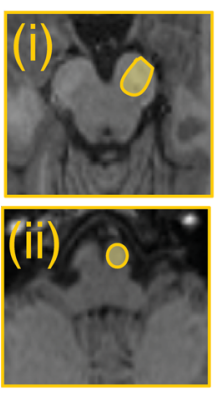

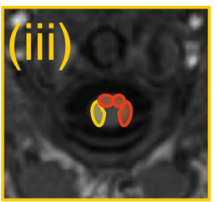

B
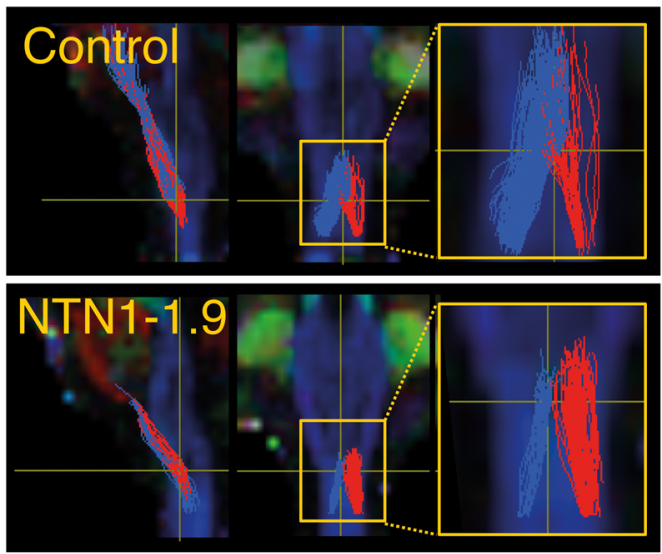

\section{C}

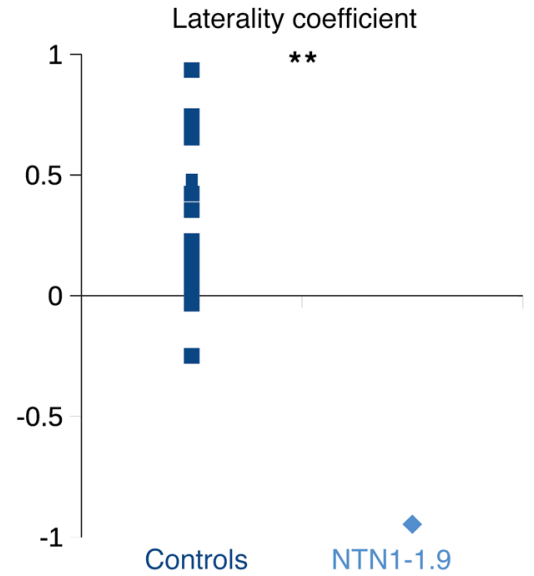

D

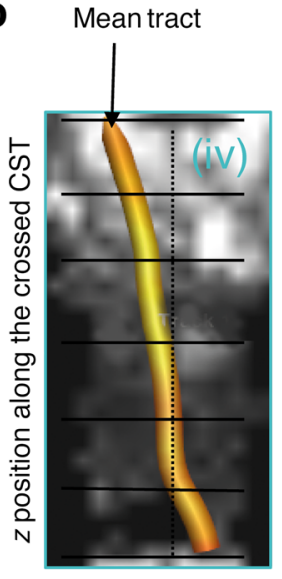

Mean FA

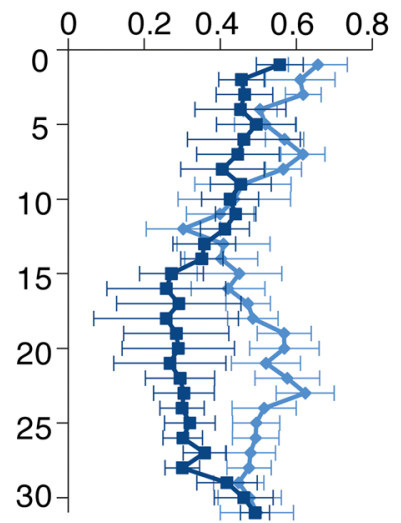

Figure 3. CST analysis using tractography. (A) Illustration of crossed (blue) and uncrossed (red) CSTs. Images show the ROI used to reconstruct the CST at the base of the pontine nuclei (i), the anterior pyramid in the upper medulla (ii), and the crossed lateral (yellow) funiculus of the upper cervical cord (iii). For example, the crossed CST from the right primary motor cortex (M1) to the left upper cervical cord was reconstructed excluding fibers reaching the left medial and the right lateral and medial funiculus (red). (B) Tractography of the CST (same color coding as in A) superimposed on the individual FA color map (sagittal views on the left, coronal views in the middle, zoom of the tracts inferior to the decussation on the right) of a control subject and an NTN1 patient (NTN1-1.9). The CST laterality coefficient, expressed as (NF crossed - NF uncrossed)/(NF crossed + NF uncrossed), was positive for the control ( 0.93 , indicating more connections in the crossed CST) and negative for the NTN1 patient (-0.94, indicating more connections in the uncrossed CST). (C) The CST laterality coefficients of 20 control subjects were compared with that of the NTN1-1.9 patient (Crawford-Howell $t$ test, ${ }^{* *} P=0.001$ ). (D) Mean FA along the crossed CST. The mean tract from the upper brainstem to the funiculus of the upper spinal cord is represented in yellow and is superimposed on the coronal view of the FA map of the control subject. Note that the $z$ axis displays the anatomical correspondence between the coronal view (left) and the graph of the mean FA values (right), indicating that the pyramidal decussation occurs between $17<z<25$. Mean FA values increased for the NTN1-1.9 patient (diamonds) compared with values for the control subject (squares) at the level of the pyramidal decussation. Error bars represent standard errors.

magnetic stimulation (TMS) to study the propagation of neural signals along the CST in 2 patients (patients 1.9 and 2.3) and 2 control subjects (age and sex matched). We found that unilateral stimulation of the primary motor cortex frequently elicited bilateral motor-evoked potentials (MEPs) in patients compared with strictly unilateral MEPs, contralateral to the stimulation, in controls (Figure 4 and Table 2). From this multimodal approach, we showed that patients with CMM due to NTN1 mutations have an increased proportion of ipsilateral CST projections.

The 3 mutated netrin-1 proteins were almost exclusively detected in the intracellular compartments. To assess the pathogenicity of the 3 variants, mutations (I518del, C601R, and C601S) were introduced into mouse and human netrin-1 cDNAs fused to an alkaline phosphatase (AP) reporter. We showed that the mutated
netrin-1-AP proteins could be detected in HEK293 cell lysates, but not in the supernatant, contrary to the WT netrin-1-AP constructs (Figure 5, A and B). Similar results were obtained using untagged human WT netrin-1 and a netrin-1 ${ }^{\mathrm{C} 601 \mathrm{R}}$ variant (Supplemental Figure $3, \mathrm{~A}$ and $\mathrm{B})$. In addition, the initial rate of reaction in the AP assay was higher in the supernatant of cells transfected with WT netrin-1-AP constructs than in the supernatant of nontransfected cells, whereas there was no difference between the nontransfected cells and cells transfected with each of the mutated netrin-1-AP constructs (Figure 5C). To further demonstrate the lack of netrin-1 in the extracellular space and quantify the expression of the different netrin-1 constructs, we conducted transfection experiments in Flp-In TRex tetracycline transactivator stable HeLa cell lines. This transfection strategy allows expression cassettes to be inte- 
A Control

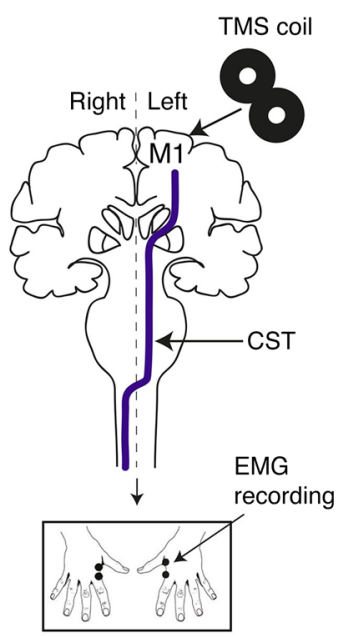

B NTN1 patient
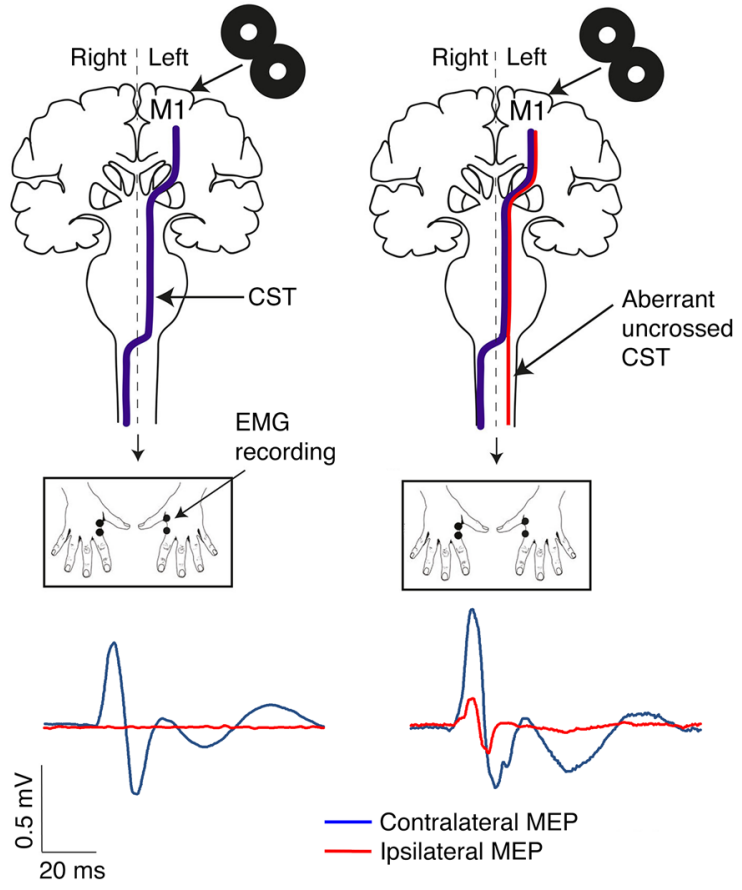

grated into only 1 predefined site in the genome, thus eliminating differences in expression levels that may arise because of different sites of integration (38). After induction by doxycycline, mutant protein levels in cell lysates were either comparable (C601S) or lower (I518del and C601R) than WT protein levels, indicating that the intracellular stability of the mutant proteins might be slightly reduced. In contrast to WT netrin-1, the mutated netrin-1 proteins could not be detected in the supernatants (Figure 5E). However, by concentrating the supernatants, we were able to detect very low levels of the 3 mutant proteins, indicating a minimal presence of the mutant proteins in the extracellular space (Figure 5F). Finally, we studied cellular localization through immunofluorescence. In the absence of doxycycline, no netrin-1 was detected (Figure 6). After induction by doxycycline, none of the constructs was detected in the nucleus (Figure 6). We found that mutant netrin-1 proteins were mostly confined to the cytoplasm (double labeling with actin), whereas WT netrin-1 was mainly detected in the extracellular compartment (Figure 7). In both HEK293 and HeLa cells, all 3 mutated proteins found in patients with CMM were almost exclusively detected in the cellular compartment, whereas WT netrin-1 was detected in both cellular and extracellular compartments.

\section{Discussion}

We have shown that heterozygous mutations in exon 7 of NTN1 caused autosomal dominant CMM in 2 unrelated families and 1 sporadic case. NTN1-CMM patients have an increased proportion of ipsilateral CST projections compared with that observed in control subjects. When expressed in HEK293 or HeLa cells, the mutated proteins were almost exclu-
Figure 4. Analysis of neural signal propagation along the CSTs using single-pulse TMS. (A and B) Schematic representation of the TMS experiments. In controls (A), unilateral stimulation of the hand area of the dominant primary motor cortex (M1) with TMS elicited contralateral MEPs only (A, blue line), whereas bilateral MEPs were observed in NTN1 patients (B, contralateral blue and ipsilateral red lines).

sively detected in the intracellular compartment, contrary to WT netrin-1. Since netrin-1 is a diffusible extracellular cue, the pathophysiology probably involves loss of function. As a consequence, CST midline axonal guidance was likely disrupted in the patients.

Given the numerous roles of netrin-1 during development and beyond, the absence of manifestations other than mirror movements is quite surprising. None of the subjects in our study had vision or hearing impairment (39), oculomotor abnormalities (40), hypogonadism (41), cardiovascular diseases (13), cancers (12), or inflammatory diseases (14). Constipation-dominant IBS and temperature regulation issues (10) have each been observed in 2 patients. The occurrence of IBS is especially interesting. Considering its high prevalence in the general population and the lack of specificity of the core IBS manifestations, it could be a coincidence. However, it is noteworthy that its onset was in infancy in our 2 patients instead of in young adulthood, as is the case in most patients with IBS. In addition, netrins and DCC have been linked to the distribution of neurons in the enteric nervous system, specifically to the migration of neurons to the submucosal plexus and to the pancreas (42). Abnormalities of the submucosal plexus are likely to affect gastrointestinal secretion of chloride and thus water, which could cause defects in motility. Submucosal neuronal abnormalities could also lead to defects in intrinsic motor reflexes, with a similar outcome. These might result in changes that are interpreted as IBS. No data were available concerning the secretion of pancreatic enzymes or hormones in our patients.

The lack of other phenotypes might be explained first by the fact that DCC has other ligands outside netrin-1 and that it also binds to other secreted netrins (43-45), cerebellin-4 (46), and other receptors such as ROBOs and UNC5s (47). Therefore, DCC mutations are expected to lead to more severe brain phenotypes than NTN1 mutations. In addition, the patients only carry heterozygous mutations, and it is therefore difficult to extrapolate from

Table 2. Frequency and amplitude of the mirror MEPs in 2 NTN1 patients

\begin{tabular}{|cccc|}
\hline Patient & Stimulated hemisphere & $\begin{array}{c}\text { Frequency of } \\
\text { ipsilateral MEPs }\end{array}$ & $\begin{array}{c}\text { Relative amplitude } \\
\text { (ipsi-/contralateral MEPs) }\end{array}$ \\
\hline 2.3 & Dominant (left) & $19 \%$ & 0.16 \\
\hline 1.9 & Nondominant (right) & $57 \%$ & 1.27 \\
\hline Controls & Dominant (left) & $17 \%$ & 0.02 \\
\hline$(n=2)$ & Nondominant (right) & $98 \%$ & 0.31 \\
\hline
\end{tabular}

The frequency of ipsilateral MEPs is the percentage of trials in which bilateral muscle responses were elicited from the unilateral stimulation of the dominant or nondominant hemisphere. 


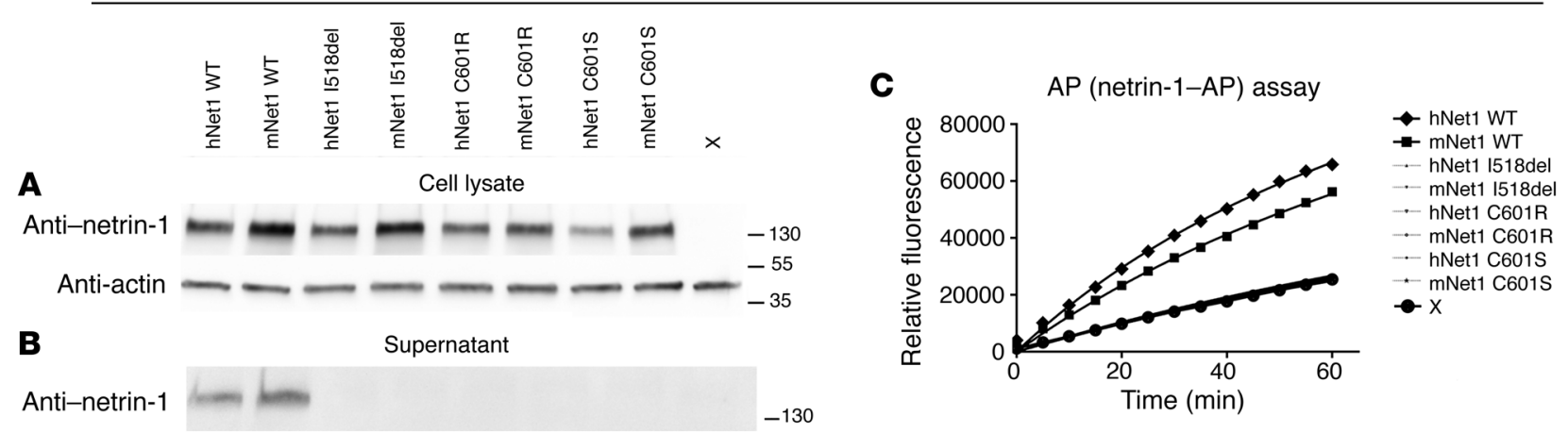

Stable HeLa lines
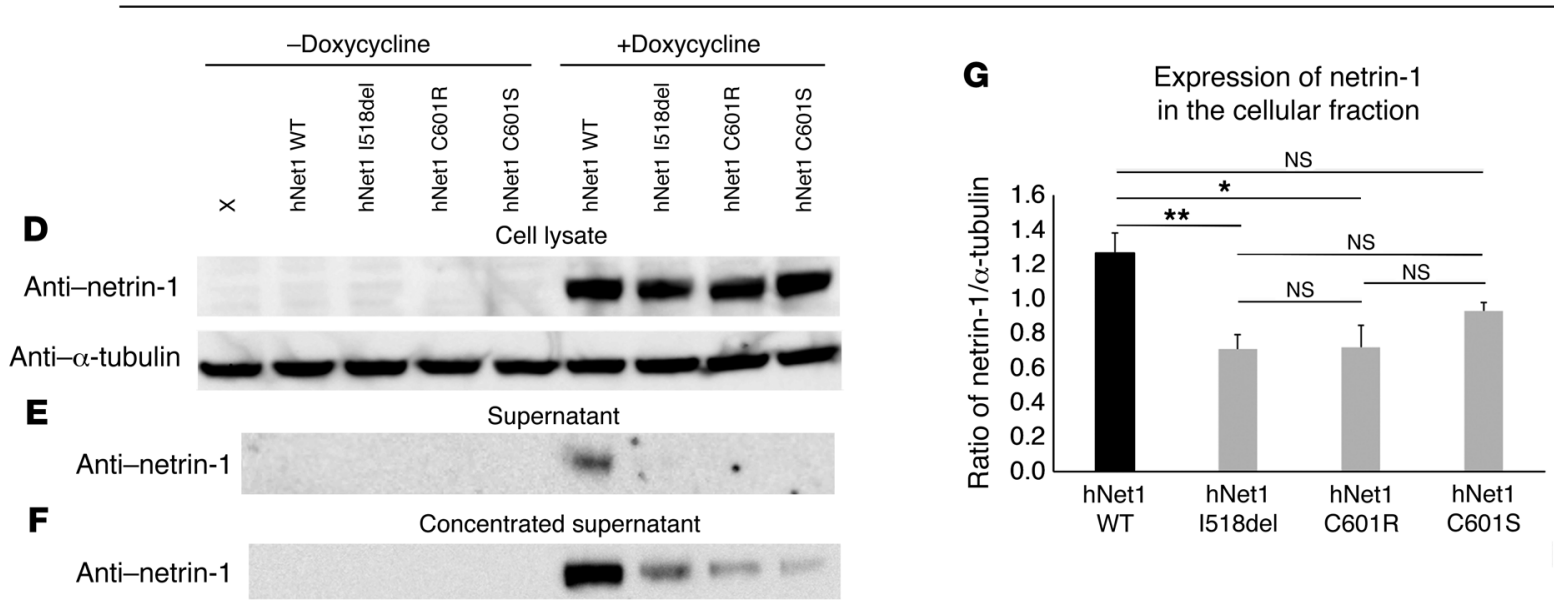

Figure 5. Expression of the WT and mutated netrin-1-AP and netrin-1 constructs. (A-C) HEK293 cells were transfected with mouse and human WT and mutated netrin-1-AP plasmids and grown for 48 hours. Western blot showed the presence of the WT and mutated proteins in total lysates at the expected molecular weight (A) but no detection of the mutated proteins in the supernatant, contrary to that seen with WT (B). AP assay of the supernatants (C) showed no difference in the initial rate of reaction between nontransfected cells $(X)$ and cells transfected with mutated netrin-1-AP plasmids, indicating that mutated netrin-1-AP levels in the supernatant were under the detection level. The experiments were replicated 3 times. The antibodies used were anti-netrin-1 (A, B, and D-F), anti-actin (A), and anti- $\alpha$-tubulin (D). Flp-In TRex tetracycline transactivator HeLa cells were transfected with the WT or 3 netrin-1 constructs harboring the NTN1 mutations (D-G). Stable cell lines were grown for 24 hours in the presence or absence of doxycycline. Western blot showed the presence of WT and mutated proteins in total lysates at the expected molecular weight in the presence of doxycycline (D) but no detection of the mutated proteins in the supernatant, contrary to that seen with WT (E). A small amount of mutated proteins could be detected in the supernatant after concentration (F). In all cases, no netrin-1 protein could be detected in the absence of doxycycline (D-F). (C) The ratio of netrin-1/ $\alpha$-tubulin was significantly reduced only for the mutated I518del and C601R forms compared with WT (1-way ANOVA $[\mathbf{F}(3,12)=6.44, P=0.008]$ followed by Bonferroni's post-hoc test, ${ }^{*} P<0.05$ and $\left.{ }^{* *} P<0.01\right)$. h, human, m, mouse; Net1, netrin-1. The experiments were replicated 3 times.

what has been described in Ntn1-null or hypomorphic-mutant mice. Other human secreted netrins (NTN2/3, NTN4, and NTN5) could partially compensate for the lack of netrin- 1 . Whether these netrins act on CST axons is unknown, and neither netrin-5-KO (45) nor netrin-4-KO (48) mice display locomotor defects.

Furthermore, penetrance was incomplete, at least in family 1. Incomplete penetrance has already been observed for RAD51 and DCC mutations $(19,21,29)$. Interestingly, despite the small number of patients, both tractography and TMS results converged to support the existence of an abnormal uncrossed CST in NTN1 patients. The presence of an abnormal uncrossed CST has also been observed in RAD51-CMM and DCC-CMM patients $(26,49)$. Altogether, these results suggest that the CST midline guidance function of netrin-1, as with RAD51 and DCC, is the most sensitive to loss of function, with abnormalities occurring when only a subfraction of the translated protein is fully functional, whereas alterations of other functions might require greater deficits.

The presence of numerous uncrossed CST fibers in NTN1 and DCC-CMM patients is consistent with findings in severe hypomorphic Ntn1-mutant mice, which show much-reduced decussation of the CST (6). These mutants also exhibit corpus callosum agenesis (7), an absence of forebrain commissures, and defects in spinal commissural axon projections (5). Ntn1 hypomorphic mutants usually die shortly after birth. Dcc-KO mice also display commissural axon guidance defects and are not viable (50). Electrophysiological recordings in embryonic spinal cord preparations showed that neuronal circuits controlling locomotion are disorganized in $D c c$ and Ntn1 mutants (51). In addition, Dcc mutants carrying the "kanga" mutation (which results in a deletion of the P3 domain in the cytoplasmic tail of DCC) have a hopping gait (6). These mice 


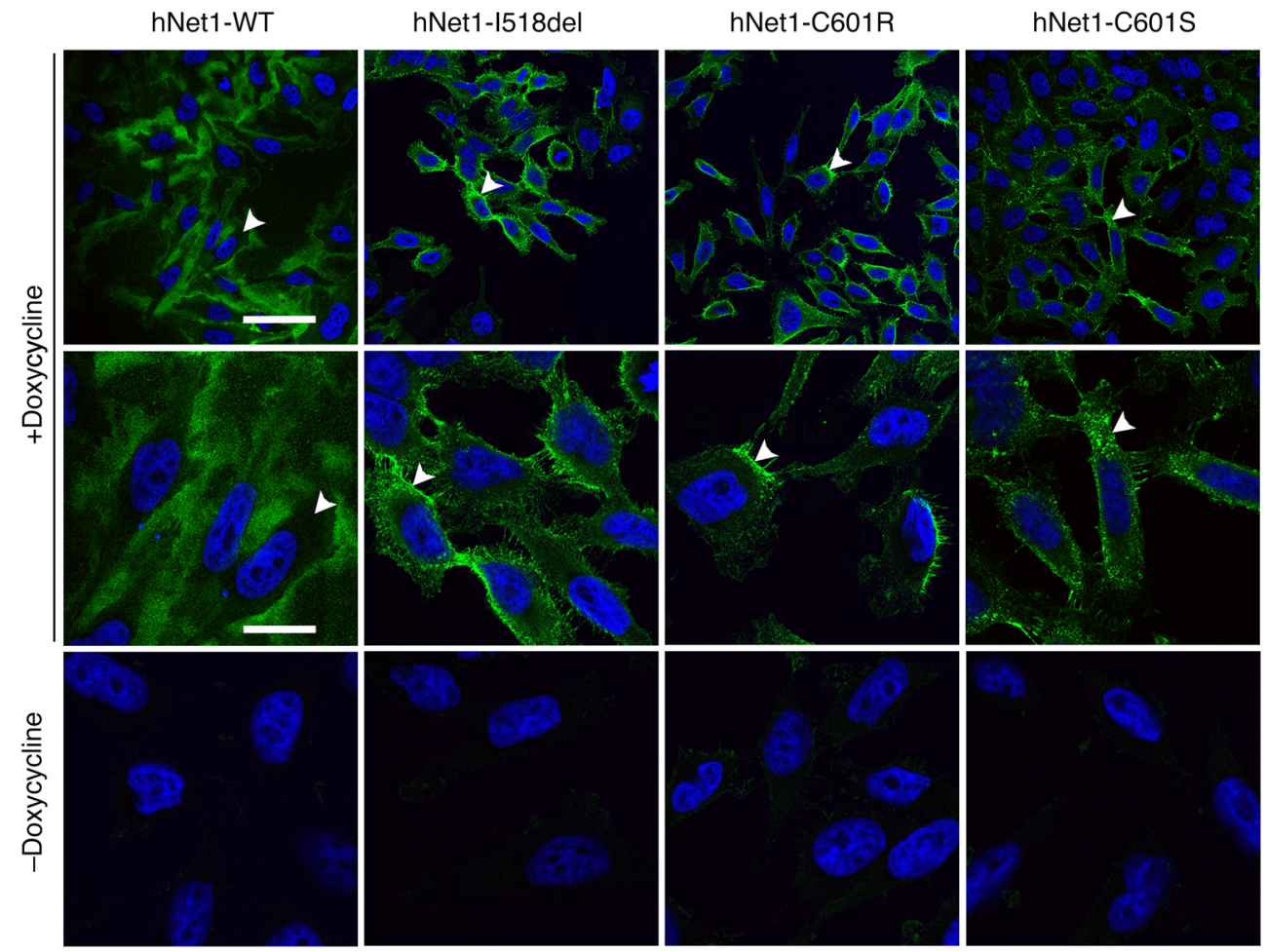

Anti-c-Myc (netrin-1-c-Myc) + Hoechst
Figure 6. Specific expression of netrin-1 after doxycycline treatment in stable HeLa cell lines. Stable HeLa cell lines (FIp-In TRex tetracycline transactivator HeLa cells transfected with the 3 netrin-1 constructs harboring the NTN1 mutations or the WT construct) were grown for 24 hours in the presence or absence of doxycycline. The experiments were replicated 3 times. Confocal microscopy (original magnification, $\times 63$ ) showed that no netrin-1 was detected in the absence of doxycycline. None of the proteins was detected in the nucleus. Hoechst staining (blue) and anti-c-Myc (green to detect netrin-1) were used. In each condition, arrowheads point to the same cell at low (first line) and high (second line) magnification. Scale bars: $60 \mu \mathrm{m}$ for the top row and $9.5 \mu \mathrm{m}$ for the bottom 2 rows. also present with corpus callosum agenesis and defects in the decussation of the CST. This is partly consistent with the abnormalities found in CMM patients that involve both altered interhemispheric inhibition and abnormal decussation of the CST (26, $27)$. The study of Ntn1-conditional KO mice $(3,10)$ might allow for a better understanding of the pathogenic underpinnings of CMM in NTN1 patients. In addition, $N t n-1^{+/-}$mice showed normal neuronal anatomy of the colon but heightened colonic inflammation in experimental colitis (52). This is in keeping with the hypothesis that NTN1 mutations may induce intestinal disease.

NTN1 mutations probably alter netrin-1 function and/or stability through deleterious structural modifications that result in a lack of netrin- 1 in the extracellular compartment. The 3 reported variants are 2 missense mutations and 1 in-frame deletion located in a single domain, the NTR domain, at the C-terminal part of the protein. The NTR domain is not necessary for secretion (53) and not required for DCC binding (54-56). The 3 mutations are predicted to be pathogenic by different algorithms (Table 1), whereas very few NTN1 variants in the ExAC database are predicted to be damaging. The DECIPHER database (DatabasE of genomiC varIation and Phenotype in Humans using Ensembl Resources, https:// decipher.sanger.ac.uk/) lists 3 deletions encompassing NTN1, including, respectively, 28, 65, and 1,299 genes that have not been associated with CMM. In the latter, the reported phenotype is severe, including, among others, developmental delay. These individuals might have CMM that has gone undetected, in the presence of more obvious comorbidities associated with the deletion. Incomplete penetrance and difficulty to diagnose CMM cloud the picture as well, as they do for potentially pathogenic alleles that are present in online databases like ExAC. The frequency of NTN1 variants predicted to be damaging was significantly higher in individuals with CMM than in the ExAC population. The 6 conserved cysteines located in the NTR module of netrin-1 form disulphide bonds that are probably very important for the structure of netrin-1 (35). The cysteine in position 601 seems to be of particular importance, as it was mutated in 2 of our 3 families. The structural model suggests that $\mathrm{C601R}$ and $\mathrm{C601S}$ mutants most likely induce the disruption of a disulfide bridge and therefore alter the folding of the NTR domain. Besides, mutation of 1 cysteine involved in a disulfide bridge leaves the other cysteine (C491) free, which is potentially highly detrimental for proper expression in the extracellular medium. In parallel, the 1518 deletion induces a deletion of 1 amino acid in a $\beta$-strand that is predicted to be deleterious and may be highly detrimental for the structure of the NTR domain. Altogether, our data strongly support the pathogenicity of these 3 variants. More demonstrative, we show that the mutated proteins are present in extremely small amounts in the extracellular space in contrast to WT netrin-1. Since netrin-1 is an extracellular cue, a decrease of its quantity in the extracellular space might explain the pathophysiology through loss of function. As mutant protein quantity was either comparable or smaller than WT proteins in the cellular compartments, the defect of netrin-1 in the extracellular space might be due to a secretion defect or a slightly reduced stability of the mutant proteins. Consistent with reports of the existence of a nucleolus localization signal in the C-terminal part of netrin-1 (57), we considered the alternative hypothesis of an abnormal relocation of the protein to the nucleus. However, our experiments showed that the mutant netrin-1 proteins were not detected in the nucleus but were located throughout the cytoplasm. The quasiabsence of mutated netrin- 1 in the extracellular space might thus be due to impairment of the secretion process or to protein instability, particularly in the extracellular space. Although the NTR, 


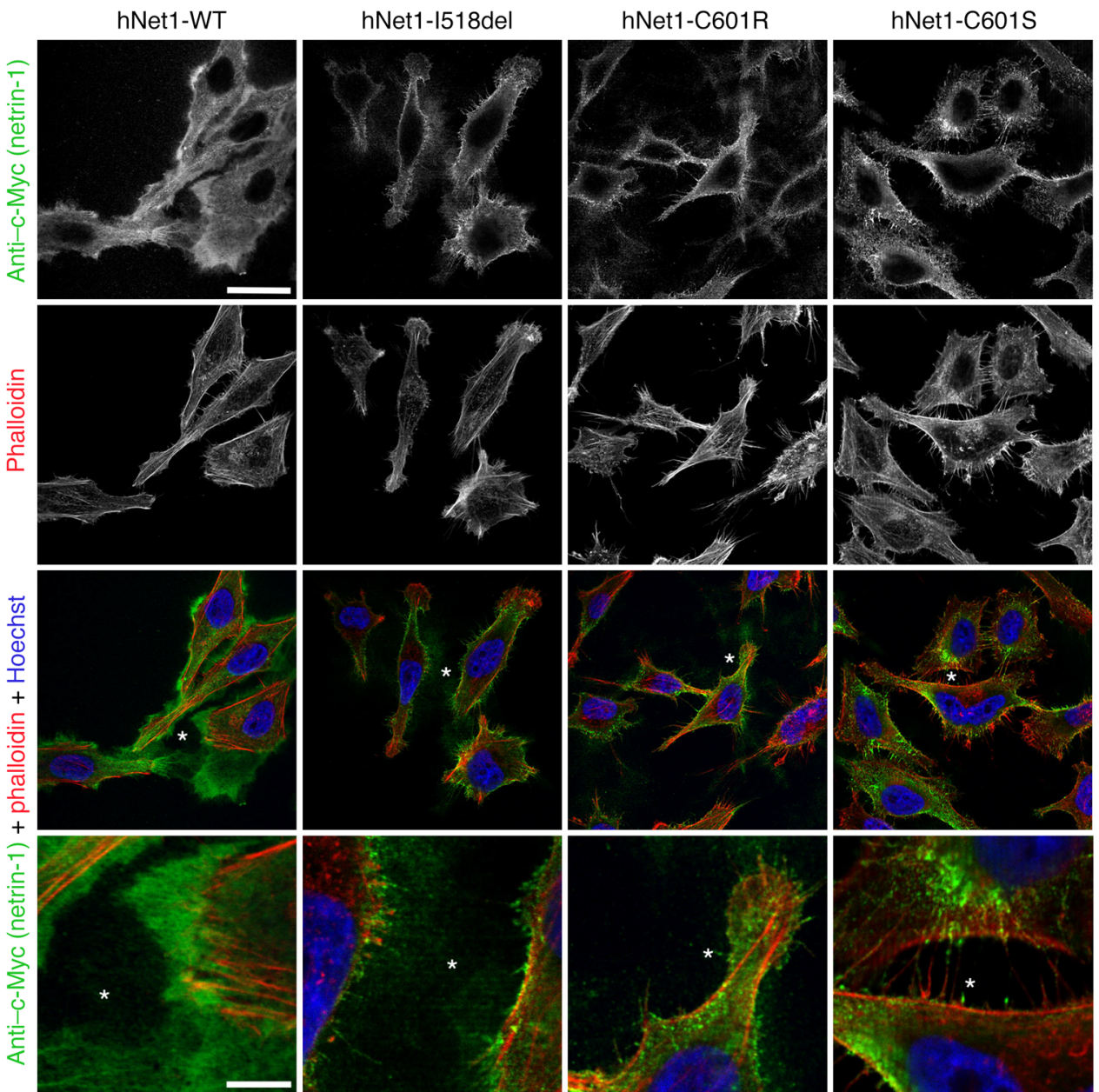

Figure 7. Localization of WT and mutated netrin-1 expressed in stable HeLa cell lines. Stable HeLa stable cell lines were grown for 24 hours in the presence of doxycycline. The experiments were replicated 3 times. Confocal microscopy (original magnification, $\times 63$ ) showed that WT netrin-1 was present in the extracellular compartment, whereas the 3 mutants were mainly confined to the cytoplasm in the presence of doxycycline. None of the constructs was detected in the nucleus. Hoechst staining (blue), phalloidin (actin, red), and anti-c-Myc (netrin-1, green) were used. For each condition, asterisks are in the same place at low (third row) and high (last row) magnification. Scale bars: $30 \mu \mathrm{m}$ in the top 3 rows and $7 \mu \mathrm{m}$ in the bottom row. per se, is thought not to be required for netrin-1 secretion (46) but might be involved in the control of the activity of metalloproteases (35), our main hypothesis is that its structural disruption might prevent the presence of secreted netrin- 1 in the extracellular space. Further studies are needed to decipher the exact mechanisms by which NTN1 mutations result in a lack of netrin-1 in the extracellular compartment. Since netrin-1 is considered a potential therapeutic target for cancer and inflammatory diseases, identification of these mechanisms might open new therapeutic avenues.

\section{Methods}

\section{Patient selection}

Data on international cases were collected over a period of 5 years in France and New Zealand. Twenty-five index cases without a mutation in either DCC or RAD51 (rearrangements being excluded in 14 of them) were selected for exome sequencing. Each patient as well as available family members underwent a standardized neurologic assessment and DNA sampling. The severity of mirror movements (MM) was scored with the Woods and Teuber scale. Familial history, MM location, associated disorders, and reported functional disability were noted.

\section{Exome sequencing and analysis}

With regard to the exome sequencing performed in France, genomic DNA was captured using Agilent in-solution enrichment methodolo- gy (SureSelect Human All Exon Kits V5; Agilent Technologies) with their biotinylated oligonucleotide probes library (SureSelect Human All Exon V5, $50 \mathrm{Mb}$; Agilent Technologies), followed by paired-end 75-base massive parallel sequencing on an Illumina HiSEQ 2000. Sequence capture, enrichment, and elution were performed according to the manufacturer's instructions and protocols, without modification except for library preparation, which was performed with the NEBNext Ultra Kit (New England BioLabs). For library preparation, 600 ng of each genomic DNA was fragmented by sonication and purified to yield fragments of 150 to 200 bp. Paired-end adaptor oligonucleotides from the NEB kit were ligated on repaired, A-tailed fragments and then purified and enriched by 8 PCR cycles. Purified libraries (1.2 $\mu \mathrm{g})$ were then hybridized to the SureSelect oligonucleotide probe capture library for 72 hours. After hybridization, washing, and elution, the eluted fraction was PCR amplified with 9 cycles and then purified and quantified by quantitative PCR (qPCR) to obtain an adequate DNA template for downstream applications. Each elution-enriched DNA sample was then sequenced on an Illumina HiSEQ 2000 as pairedend 75-base reads. Image analysis and base calling were performed using an Illumina Real Time Analysis (RTA 2.1.3) system with default parameters. Bioinformatics analysis of sequencing data was based on the Illumina pipeline (CASAVA 1.8.2). CASAVA performs alignment of a sequencing run to a reference genome (hg19), calls the SNPs on the basis of the allele calls and read depth, and detects variants (SNPs and indels). The alignment algorithm used was ELANDv2 (CASAVA), 
which performs multiseed and gapped alignments. Only the positions included in the bait coordinates were conserved. Genetic variation annotation was realized from the IntegraGen in-house pipeline. It consists of gene annotations (RefSeq, https://www.ncbi.nlm.nih.gov/ refseq/) and known polymorphisms (dbSNP 132, Single Nucleotide Polymorphism Database, https://www.ncbi.nlm.nih.gov/projects/ SNP/; 1000 Genomes, http://www.1000genomes.org/; and EVS, http://evs.gs.washington.edu/EVS/), followed by a mutation characterization (exonic, intronic, silent, nonsense). For each position, the exomic frequencies (Homo and HTZ) were determined from all the exomes already sequenced at IntegraGen, and the exome results were provided by 1000 Genomes, EVS, and HapMap (haplotype map of the human genome, ftp://ftp.ncbi.nlm.nih.gov/hapmap/) algorithms. The minimum average depth on the exome was approximately $50 \times$. When coverage was below $50 \times$ for an exon and/or bp in NTN1 (which was the case for exon 6 and the first bp of exon 2 in most patients), the analysis was completed by Sanger sequencing.

Concerning the exome sequencing performed in New Zealand, genomic DNA was collected and extracted from saliva samples using Oragene DNA kits (DNA Genotek Inc.). Exome sequencing was provided by Otogenetics Corporation using Agilent SureSelect Human All Exon V4 or Agilent SureSelect Human All Exon V5+UTR for capture and Illumina sequencing platforms for data generation (paired end, 100 cycles). Sequence alignment was performed with the BurrowsWheeler Aligner (BWA) mem using human genome assembly GRCh37 as the reference. Further processing was done with Picard tools and the Genome Analysis Toolkit following the Broad Institute's best practices guidelines (https://www.broadinstitute.org/gatk/) to produce called variants with HaplotypeCaller. Estimates for population allele frequencies of individual variants were obtained from the National Heart, Lung, and Blood Institute's (NHLBI) Gene Ontology (GO) Exome Sequencing Project (ESP6500) database, the 1000 Genomes Project, and the ExAC (http://exac.broadinstitute.org/) database. The predicted effect of individual variants on gene function was identified through annotation with SnpEff (58).

We then conducted the analysis with Variant Studio (Illumina). Variants present at an allele frequency greater than $0.1 \%$ in the general population (1000 Genomes and EVS databases), synonymous variants, and intronic variants that were not predicted to alter splicing were discarded. Variants with a read depth below $10 \times$ in the French exomes and $5 \times$ in the New Zealand exomes were also filtered out. A lower threshold was chosen for the New Zealand exomes, as coverage was poorer because the DNA was extracted from saliva rather than blood.

We listed all sequence variants detected in NTN1 in 60,706 controls on the ExAC algorithm. We used Fisher's exact test to compare the frequency of truncating and rare (frequency $<1 \%$ ) missense variants predicted to be pathogenic by either the PolyPhen-2 or SIFT algorithm in our patients and control subjects.

\section{Sanger sequencing and quantitative multiplex PCR of short fluorescent fragments}

The coding and flanking intronic regions of NTN1 were amplified using the primers listed in Supplemental Table 6. Forward and reverse sequencing reactions were performed with the Big Dye Terminator Cycle Sequencing Ready Reaction Kit (PE Applied Biosystems), and the products were analyzed on an $\mathrm{ABI} 3730$ automated sequencer (PE
Applied Biosystems). Quantitative multiplex PCR of short fluorescent fragments (QMPSF) analysis was performed for all exons of NTN1 in 14 patients, and results were analyzed using GeneMapper analysis software, version 4.0 (Applied Biosystems).

\section{Structural analysis}

On the basis of the alignment obtained using the HHpred method (59), a structural model was generated using Rosetta comparative modeling software (34). The template structure used to build the model is the complement C3C structure sharing $23 \%$ of sequence identity with the NTR domain of netrin-1 (Protein Data Bank [PDB] code 2a74).

\section{Site-directed mutagenesis}

To generate human and mouse netrin-1 AP fusion proteins in the C-terminal, human and mouse NTN1 cDNAs were amplified by PCR and cloned in pAP-Tag-5 (GenHunter, no. Q202) between NheI and BglII sites. Plasmids encoding human netrin- 1 and mouse netrin-1 were provided by Patrick Mehlen (Centre de Recherche en Cancérologie de Lyon [CRCL], Lyon, France). Mutations were introduced by site-directed mutagenesis (QuikChange II Site-Directed Mutagenesis Kit; Agilent Technologies) with primers (listed in Supplemental Table 7) containing the mutations and verified by Sanger sequencing. Clones were then selected and purified (ZR Plasmid Miniprep Classic from Zymo Research and NucleoBondXtra Midi/Maxi from MachereyNagel). We ensured that no other mutation was introduced by sequencing the entire cDNA of NTN1.

\section{Stable cell lines}

Flp-In T-REx HeLa cells were provided by Stephen Taylor (University of Manchester, Manchester, United Kingdom) and cultured (not authenticated, tested for mycoplasma contamination with a negative result) as described previously (38), and pcDNA5.2/FRT/TO-6cMyc was provided by Alain Joliot (Collège de France, Paris, France). NTN1hs cDNA was amplified with Phusion DNA polymerase (Thermo Fisher Scientific) and cloned into pcDNA5.2/FRT/TO-6cMyc between the NheI and BsrGI sites using the NEBuilder HiFi DNA Assembly Cloning Kit (New England BioLabs). Mutations were introduced by site-directed mutagenesis using the QuikChange Lightning Site-Directed Mutagenesis Kit with primers (listed in Supplemental Table 7) containing the mutations and verified by Sanger sequencing. Plasmids pcDNA5.2/FRT/TO/NTN1-6cMyc (WT and mutants) were cotransfected with pOG44, a plasmid expressing the Flp recombinase (Invitrogen, Thermo Fisher Scientific), into Flp-In T-REx HeLa cells using Lipofectamine 2000 Reagent (Invitrogen, Thermo Fisher Scientific) according to the manufacturer's instructions. Cells were selected in $5 \mu \mathrm{g} / \mathrm{ml}$ blasticidin (Life Technologies, Thermo Fisher Scientific) and $250 \mu \mathrm{g} / \mathrm{ml}$ hygromycin B (Sigma-Aldrich). Netrin-1 expression was induced by the addition of $1 \mu \mathrm{g} / \mathrm{ml}$ doxycycline (SigmaAldrich) for 24 hours.

\section{Immunofluorescence labeling}

Twenty-four hours after induction, cells grown on coverslips were fixed with $4 \%(\mathrm{v} / \mathrm{v})$ paraformaldehyde for fifteen minutes, permeabilized in $0.1 \%$ Triton X-100/PBS for ten minutes, and blocked in PBS containing 1\% BSA (Sigma-Aldrich). Cells were incubated for 45 minutes with Phalloidin-TRITC (Sigma-Aldrich) to detect the cytoplasm by labeling filamentous actin and then overnight at $4^{\circ} \mathrm{C}$ with 
monoclonal anti-c-Myc antibody (1:100; Santa Cruz Biotechnology Inc.; sc-40, RRID:AB_627268) (60) to detect netrin-1. Alexa Fluor 488 donkey anti-mouse (1:1,000; Jackson ImmunoResearch Laboratories; 715-545-150) was used as a secondary antibody. Nuclei were counterstained with Hoechst (Sigma-Aldrich). Noninduced cells were treated in the same way. Cellular localization of proteins was examined by confocal microscopy and replicated 3 times.

\section{Western blot analysis}

HEK293 cells (cell line from human embryonic kidney, Ad5 DNA transformed; ATCC, not authenticated, tested for mycoplasma contamination with a negative result) were transfected with pAPtag5, pAPtag5-netrin-1 human or pAPtag5-netrin-1 mouse, WT, and mutated plasmids using Lipofectamine 2000 Reagent (Invitrogen, Thermo Fisher Scientific) and grown for 48 hours. Cells were separated from medium by centrifugation, and total cellular proteins were extracted in lysis buffer (10 mM HEPES [pH 7], $100 \mathrm{mM} \mathrm{NaCl}$, 2 mM EDTA, 0.5\% NP-40).

For the experiments on stable lines, 24 hours after induction, supernatants were collected and centrifuged to pellet cell debris. The resulting supernatants were directly used for protein analysis or concentrated using Amicon Ultra-15 centrifugal filters (Ultracel 30K; EMD Millipore). Proteins from cells were extracted in lysis buffer (150 $\mathrm{mM} \mathrm{NaCl}, 25$ mM Tris-HCl [pH 7.5], 5\% glycerol, 1\% NP-40, 0.5\% sodium deoxycholate, $1 \mathrm{mM}$ EDTA and protease inhibitor cocktail).

The samples were then loaded on $4 \%$ to $15 \%$ Mini-PROTEAN TGX Tris-glycine buffer SDS-PAGE and transferred onto a $0.2-\mu \mathrm{m}$ Trans-Blot Turbo nitrocellulose membrane (Bio-Rad). Membranes were blocked for 1 hour at room temperature in $1 \times$ TBS $(10 \mathrm{mM}$ Tris$\mathrm{HCl}$ [pH 8], $150 \mathrm{mM} \mathrm{NaCl}$ ) supplemented with 5\% (w/v) dried skim milk powder. Primary antibody incubation was carried out overnight at $4^{\circ} \mathrm{C}$. The presence of netrin- 1 in total lysates and in the supernatants at the expected molecular weight was confirmed by Western blotting with anti-netrin-1 antibodies (1:500; R\&D Systems; catalog MAB1109, RRID:AB_2154710) (61). Anti-actin (1:1,000; EMD Millipore; catalog MAB1501, RRID:AB_2223041) (62) and anti- $\alpha$-tubulin (1:4,000; SigmaAldrich; catalog T5168, RRID:AB_477579) (63) antibodies were used as controls. The secondary HRP-coupled antibodies used were donkey anti-rat for detection of netrin-1, goat anti-mouse for actin (Jackson ImmunoResearch Laboratories; 712-036-153 and 115-035-003, respectively), and sheep anti-mouse for $\alpha$-tubulin (GE Healthcare). In between and after antibody incubations, membranes were extensively washed in TBS containing $2.5 \%$ Tween-20 (TBS-T). Western blots were visualized using the ECL method (Pierce ECL Western Blotting Substrate, and to better detect netrin-1, the SuperSignal West Femto Maximum Sensitivity Substrate; Thermo Fisher Scientific). To exclude an effect of the AP tag on netrin-1 secretion, we removed it from human WT and C601R-mutated netrin-1-AP plasmids by BglII/ $\mathrm{XbaI}$ digestion. We then conducted the same experiments described above. Western blot quantification for 3 independent experiments was performed using Image J software (NIH).

\section{Determination of AP activity in the supernatant}

Forty-eight hours after transfection of HEK293 cells, supernatants were cleared of cells by centrifugation. Supernatants (100 $\mu \mathrm{l}, 1: 100)$ were dispended in 96-well plates. Three replicates were tested for each sample. Plates were incubated for 1 hour with $100 \mu \mathrm{l}$ AP sub- strates according to the manufacturer's instructions (AttoPhos AP Fluorescent Substrate System; Promega). Fluorescence intensity was measured at excitation 430/emission 570, every 5 minutes, using TECAN Infinite M1000. Kinetics activity was calculated using GraphPad Prism 7 (GraphPad Software).

\section{MRI}

MRI data were acquired using a Siemens VERIO 3T scanner with a 32-channel head coil. The MRI protocol included anatomical 3D T1-weighted magnetization-prepared rapid gradient echo (MP RAGE) imaging (repetition time, $\mathrm{TR}=2.3 \mathrm{~s}$; echo time, $\mathrm{TE}=4.18 \mathrm{~ms}$; flip angle $=9^{\circ}$; inversion time, $\mathrm{TI}=900 \mathrm{~ms}$; voxel size $=1 \times 1 \times 1 \mathrm{~mm}^{3}$; 176 slices) and spin-echo echo-planar diffusion tensor imaging (TR $=10 \mathrm{~s}, \mathrm{TE}=87 \mathrm{~ms}$, voxel size $=2 \times 2 \times 2 \mathrm{~mm}^{3}, 60$ slices, 60 gradient-encoded directions, with a b-value of $1,500 \mathrm{~s} / \mathrm{mm}^{2}$ and 11 nondiffusion-weighted volumes).

\section{Tractography analysis}

Tractography analysis was performed for 1 patient (patient 1.9) and 20 control subjects.

Diffusion images were preprocessed according to previous methods (64-66) and processed for probabilistic diffusion tractography with MRtrix (http://www.mrtrix.org/). Raw diffusion-weighted data were corrected for motion and geometric distortions secondary to eddy currents using a registration technique based on the geometric model of distortions (67). The fiber orientation distribution function (ODF) was estimated using the constrained spherical deconvolution (CSD) method in MRtrix $(66,68)$. The sufficient angular resolution allowed high-order fiber orientation estimation algorithms (68-70). The ODF information obtained from CSD was used with a suitable fiber-tracking algorithm to infer connectivity of crossing fibers. We used a probabilistic streamlines algorithm with the entire ODF as a probability density function (ODF threshold $=0.1$; step size $=0.2 \mathrm{~mm}$ as $1 / 10$ of the voxel size; radius of curvature $=1 \mathrm{~mm}$; up-sampling of diffusion-weighted images [DWI], data to $1 \mathrm{~mm}$ ). In the native individual space, we performed a seed-to-target analysis from regions of interest (ROI) defined along the CST (see ROI section in ref. 26). These regions included the anterior bundle of the CST in the upper part of the brainstem, the lower part of the brainstem, and the lateral horn of the spinal cord (see Figure 3A). We used the following probabilistic tractography algorithm: the number of fibers connecting a seed voxel to a target voxel was calculated by sampling 1 million draws for each fiber connecting the seed to the target. The CST tracts (the normally crossed CST and the abnormally uncrossed CST) were reconstructed for each subject. The normally uncrossed CST passes through the anterior medial horn of the spinal cord. After the tracts were reconstructed, 2 complementary measures were considered. First, the number of fibers reaching the target at the level of the lateral horn of the spinal cord (the crossed and uncrossed CST separately) was calculated. We analyzed the proportion of crossed versus uncrossed portions of the CST using a laterality coefficient (NF crossed - NF uncrossed)/ (NF crossed + NF uncrossed) (NF, number of fibers, as in ref. 71). Ratio values closer to 1 indicated more crossed than uncrossed CST fibers, whereas ratio values closer to -1 indicated more uncrossed than crossed CST. Second, mean FA values were measured along the CST. Along-tract measures of FA were obtained on the basis of B-spline resampling of the fibers and averaging the FA values for each individ- 
ual fiber at the same location (elastic model with 30 points in space at analogous anatomical locations in each individual) (72). The upper location was defined at the ROI of the upper brainstem, and the lower location was defined at the level of the ROI of the lateral horn of the spinal cord. Mean FA values were calculated at each point of the mean fiber along the $z$ axis to check for local differences at specific points of the tract. To determine whether the laterality coefficient of the patient was different from that of the 20 control subjects, we performed a Crawford-Howell $t$ test (73).

\section{Electrophysiological experiments}

Electrophysiological experiments were conducted in 2 patients (patients 1.9 and 2.3).

Electromyography recordings. Electromyographic (EMG) signals were recorded bilaterally from the first dorsal interosseous (FDI) muscle (active electrode over the motor point and reference electrode on the metacarpophalangeal joint), with disposable surface $\mathrm{Ag} / \mathrm{AgCl}$ electrodes placed $3 \mathrm{~cm}$ apart on the muscle belly.

Single-pulse TMS and corticospinal excitability. MEPs induced by single monophasic pulses delivered with a TMS device connected to a figure-of-eight coil (Magstim) were recorded from EMG signals. TMS pulses were applied over the cortical representations (hot spots) of the FDI muscles in M1 (primary motor cortex). The resting motor threshold was defined as the minimum stimulus intensity that resulted in MEPs of $50 \mu \mathrm{V}$ in at least 5 of 10 trials. Thirty to sixty MEPs evoked by a calibrated stimulation (1.2 $-1.3 \times$ resting motor threshold) of the dominant and nondominant hemispheres were recorded bilaterally in the FDI muscle to compare the frequency, latency, and amplitude of the normal contralateral MEPs with those of potential mirror MEPs recorded in the hand ipsilateral to the stimulation site.

\section{Statistics}

Data were analyzed with SPSS statistical software, version 24.0 (IBM). Normality in variable distributions and homogeneity of variances across groups were assessed with Shapiro-Wilk and Levene tests, respectively. Variables that passed the normality test were analyzed using a 1-way ANOVA followed by Bonferroni's post-hoc test for comparisons or by a Crawford-Howell $t$ test for comparison of patients with control subjects. A $P$ value of less than 0.05 was used as a cutoff for statistical significance.

\section{Study approval}

In France, the study was approved by the ethics committee of the Hôpital de la Pitié-Salpêtrière and the Comités de Protection des Personnes (CPP), Ile-de-France 6, Paris. In New Zealand, all procedures were approved by the Health and Disability Ethics Committees, Multi-region Ethics Committee of New Zealand. Written informed consent was obtained from all patients (or parents of minors) before genetic analyses. All experiments were performed according to the respective approved protocols.

\section{Author contributions}

AM, AC, ID, OT, C. Dubacq, ER, and DM wrote and revised the manuscript. ER, DM, and ID designed and supervised the study. AM, EAF, OT, TCO, YZ, RJMG, QW, SPR, CG, C. Depienne, MS, CLJ, C. Dubacq, FR, JCL, MPM, RG, JA, CF, MD, MV, ID, GAR, and $\mathrm{AB}$ acquired, analyzed, and interpreted data.

\section{Acknowledgments}

The authors would like to thank the patients for their participation in this study; the DNA and cell bank of the ICM (in particular Christelle Dussert and Sylvie Forlani) for DNA extraction; Stephen Taylor (University of Manchester, Manchester, United Kingdom); and Alain Joliot (Collège de France, Paris, France) for providing the reagents for the stable cell line studies; and the ExAC and other groups that provided exome variant data for comparison. The work undertaken in New Zealand was supported by a Royal Society of New Zealand Marsden Fund Grant. The work undertaken in France was supported by the Fondation Desmarest, Merz-Pharma, Elivie, the Journées de Neurologie de Langue Française (JNLF), and Djillali Mehri. This work was also supported by a grant from the Agence Nationale de la Recherche (ANR) (ANR-14-CE13-0004-01) and was performed within the framework of LABEX LIFESENSES (ANR-10-LABX-65,) supported by French state funds managed by the ANR within the Investissements d'Avenir program (ANR-11-IDEX-0004-02).

Address correspondence to: Isabelle Dusart, Université Pierre et Marie Curie (UPMC), Neuroscience Paris Seine, Bat B, 6eme, Case 16, 9 Quai Saint Bernard, 75005 Paris, France. Phone: 331.44.27.21.29; Email: isabelle.dusart@upmc.fr.
1. Kennedy TE, Serafini T, de la Torre JR, TessierLavigne M. Netrins are diffusible chemotropic factors for commissural axons in the embryonic spinal cord. Cell. 1994;78(3):425-435.

2. Chédotal A. Further tales of the midline. Curr Opin Neurobiol. 2011;21(1):68-75.

3. Dominici C, et al. Floor-plate-derived netrin-1 is dispensable for commissural axon guidance. Nature. 2017;545(7654):350-354.

4. Varadarajan SG, et al. Netrin1 produced by neural progenitors, not floor plate cells, is required for axon guidance in the spinal cord. Neuron. 2017;94(4):790-799.e3.

5. Serafini T, et al. Netrin-1 is required for commissural axon guidance in the developing vertebrate nervous system. Cell. 1996;87(6):1001-1014.

6. Finger JH, Bronson RT, Harris B, Johnson K, Przyborski SA, Ackerman SL. The netrin 1 receptors
Unc5h3 and Dcc are necessary at multiple choice points for the guidance of corticospinal tract axons. J Neurosci. 2002;22(23):10346-10356

7. Fothergill T, et al. Netrin-DCC signaling regulates corpus callosum formation through attraction of pioneering axons and by modulating Slit2-mediated repulsion. Cereb Cortex. 2014;24(5):1138-1151.

8. Keino-Masu K, et al. Deleted in Colorectal Cancer (DCC) encodes a netrin receptor. Cell. 1996;87(2):175-185.

9. Goldman JS, et al. Netrin-1 promotes excitatory synaptogenesis between cortical neurons by initiating synapse assembly. J Neurosci. 2013;33(44):17278-17289.

10. Brunet I, et al. Netrin-1 controls sympathetic arterial innervation. J Clin Invest. 2014;124(7):3230-3240.
11. Tepavčević V, et al. Early netrin-1 expression impairs central nervous system remyelination. Ann Neurol. 2014;76(2):252-268.

12. Mehlen P, Delloye-Bourgeois C, Chédotal A. Novel roles for Slits and netrins: axon guidance cues as anticancer targets? Nat Rev Cancer. 2011;11(3):188-197.

13. Bongo JB, Peng DQ. The neuroimmune guidance cue netrin-1: a new therapeutic target in cardiovascular disease. J Cardiol. 2014;63(2):95-98.

14. Ramesh G. Role of Netrin-1 beyond the brain: from biomarker of tissue injury to therapy for inflammatory diseases. Recent Pat Biomark. 2012;2(3):202-208.

15. Leslie EJ, et al. A multi-ethnic genome-wide association study identifies novel loci for non-syndromic cleft lip with or without cleft palate on 2p24.2, 17q23 and 19q13. Hum Mol Genet. 
2016;25(13):2862-2872.

16. Wetzel-Smith MK, et al. A rare mutation in UNC5C predisposes to late-onset Alzheimer's disease and increases neuronal cell death. Nat Med. 2014;20(12):1452-1457.

17. Marsh AP, et al. Mutations in DCC cause isolated agenesis of the corpus callosum with incomplete penetrance. Nat Genet. 2017;49(4):511-514.

18. Jamuar SS, et al. Biallelic mutations in human DCC cause developmental split-brain syndrome. Nat Genet. 2017;49(4):606-612.

19. Srour M, et al. Mutations in DCC cause congenital mirror movements. Science. 2010;328(5978):592.

20. Depienne C, et al. A novel DCC mutation and genetic heterogeneity in congenital mirror movements. Neurology. 2011;76(3):260-264.

21. Méneret A, et al. Congenital mirror movements: mutational analysis of RAD51 and DCC in 26 cases. Neurology. 2014;82(22):1999-2002.

22. Meneret A, Trouillard O, Depienne C, Roze E. Congenital mirror movements. In: Pagon RA, et al., eds. GeneReviews(R). Seattle, Washington, USA; 1993.

23. Bonnet C, Roubertie A, Doummar D, BahiBuisson N, Cochen de Cock V, Roze E. Developmental and benign movement disorders in childhood. Mov Disord. 2010;25(10):1317-1334.

24. Meneret A, Trouillard O, Brochard V, Roze E. Congenital mirror movements caused by a mutation in the DCC gene. Dev Med Child Neurol. 2015;57(8):776.

25. Méneret A, Welniarz Q, Trouillard O, Roze E. Congenital mirror movements: from piano player to opera singer. Neurology. 2015;84(8):860.

26. Gallea C, et al. RAD51 deficiency disrupts the corticospinal lateralization of motor control. Brain. 2013;136(Pt 11):3333-3346.

27. Galléa C, Popa T, Billot S, Méneret A, Depienne C, Roze E. Congenital mirror movements: a clue to understanding bimanual motor control. J Neurol. 2011;258(11):1911-1919.

28. Welniarz Q, Dusart I, Gallea C, Roze E. One hand clapping: lateralization of motor control. Front Neuroanat. 2015;9:75.

29. Depienne C, et al. RAD51 haploinsufficiency causes congenital mirror movements in humans. Am J Hum Genet. 2012;90(2):301-307.

30. Ahmed I, et al. Identification of a homozygous splice site mutation in the dynein axonemal light chain 4 gene on 22q13.1 in a large consanguineous family from Pakistan with congenital mirror movement disorder. Hum Genet. 2014;133(11):1419-1429.

31. Méneret A, Trouillard O, Vidailhet M, Depienne C, Roze E. Congenital mirror movements: no mutation in DNAL4 in 17 index cases. J Neurol. 2014;261(10):2030-2031.

32. Glendining KA, Markie D, Gardner RJ, Franz EA, Robertson SP, Jasoni CL. A novel role for the DNA repair gene Rad51 in Netrin-1 signalling. Sci Rep. 2017;7:39823.

33. Franz EA, et al. Congenital mirror movements: phenotypes associated with DCC and RAD51 mutations. J Neurol Sci. 2015;351(1-2):140-145.

34. Song Y, et al. High-resolution comparative modeling with RosettaCM. Structure. 2013;21(10):1735-1742.

35. Bányai L, Patthy L. The NTR module: domains of netrins, secreted frizzled related proteins, and type I procollagen C-proteinase enhancer protein are homologous with tissue inhibitors of metalloproteases. Protein Sci. 1999;8(8):1636-1642.

36. Chong JM, Uren A, Rubin JS, Speicher DW. Disulfide bond assignments of secreted Frizzledrelated protein-1 provide insights about Frizzled homology and netrin modules. J Biol Chem. 2002;277(7):5134-5144.

37. Liepinsh E, Banyai L, Pintacuda G, Trexler M, Patthy L, Otting G. NMR structure of the netrinlike domain (NTR) of human type I procollagen $\mathrm{C}$-proteinase enhancer defines structural consensus of NTR domains and assesses potential proteinase inhibitory activity and ligand binding. JBiol Chem. 2003;278(28):25982-25989.

38. Tighe A, Johnson VL, Taylor SS. Truncating APC mutations have dominant effects on proliferation, spindle checkpoint control, survival and chromosome stability. J Cell Sci. 2004; 117(Pt 26):6339-6353.

39. Gillespie LN, Marzella PL, Clark GM, Crook JM. Netrin-1 as a guidance molecule in the postnatal rat cochlea. Hear Res. 2005;199(1-2):117-123.

40. Burgess RW, Jucius TJ, Ackerman SL. Motor axon guidance of the mammalian trochlear and phrenic nerves: dependence on the netrin receptor Unc5c and modifier loci. J Neurosci. 2006;26(21):5756-5766.

41. Murakami S, Ohki-Hamazaki H, Watanabe K, Ikenaka K, Ono K. Netrin 1 provides a chemoattractive cue for the ventral migration of $\mathrm{GnRH}$ neurons in the chick forebrain. J Comp Neurol. 2010;518(11):2019-2034.

42. Jiang Y, Liu MT, Gershon MD. Netrins and DCC in the guidance of migrating neural crest-derived cells in the developing bowel and pancreas. Dev Biol. 2003;258(2):364-384.

43. Qin S, Yu L, Gao Y, Zhou R, Zhang C. Characterization of the receptors for axon guidance factor netrin- 4 and identification of the binding domains. Mol Cell Neurosci. 2007;34(2):243-250.

44. Wang H, Copeland NG, Gilbert DJ, Jenkins NA, Tessier-Lavigne M. Netrin-3, a mouse homolog of human NTN2L, is highly expressed in sensory ganglia and shows differential binding to netrin receptors. J Neurosci. 1999;19(12):4938-4947.

45. Garrett AM, et al. Analysis of Expression Pattern and Genetic Deletion of Netrin5 in the Developing Mouse. Front Mol Neurosci. 2016;9:3.

46. Haddick PC, et al. Defining the ligand specificity of the deleted in colorectal cancer (DCC) receptor. PLoS ONE. 2014;9(1):e84823.

47. Stein E, Tessier-Lavigne M. Hierarchical organization of guidance receptors: silencing of netrin attraction by slit through a Robo/DCC receptor complex. Science. 2001;291(5510):1928-1938.

48. Hayano Y, et al. Netrin-4 regulates thalamocortical axon branching in an activitydependent fashion. Proc Natl Acad Sci U S A. 2014;111(42):15226-15231.

49. Welniarz Q, et al. Non cell-autonomous role of DCC in the guidance of the corticospinal tract at the midline. Sci Rep. 2017;7(1):410.

50. Fazeli A, et al. Phenotype of mice lacking functional Deleted in colorectal cancer (Dcc) gene. Nature. 1997;386(6627):796-804.

51. Rabe Bernhardt N, Memic F, Gezelius H, Thiebes
AL, Vallstedt A, Kullander K. DCC mediated axon guidance of spinal interneurons is essential for normal locomotor central pattern generator function. Dev Biol. 2012;366(2):279-289.

52. Aherne CM, et al. Neuronal guidance molecule netrin-1 attenuates inflammatory cell trafficking during acute experimental colitis. Gut. 2012;61(5):695-705.

53. Kruger RP, Lee J, Li W, Guan KL. Mapping netrin receptor binding reveals domains of Unc5 regulating its tyrosine phosphorylation. J Neurosit. 2004;24(48):10826-10834.

54. Finci LI, et al. The crystal structure of netrin-1 in complex with DCC reveals the bifunctionality of netrin-1 as a guidance cue. Neuron. 2014;83(4):839-849.

55. Xu K, et al. Neural migration. Structures of netrin-1 bound to two receptors provide insight into its axon guidance mechanism. Science. 2014;344(6189):1275-1279.

56. Grandin M, et al. Structural decoding of the Netrin-1/UNC5 interaction and its therapeutical implications in cancers. Cancer Cell. 2016;29(2):173-185.

57. Delloye-Bourgeois C, et al. Nucleolar localization of a netrin-1 isoform enhances tumor cell proliferation. Sci Signal. 2012;5(236):ra57.

58. Cingolani P, et al. A program for annotating and predicting the effects of single nucleotide polymorphisms, SnpEff: SNPs in the genome of Drosophila melanogaster strain w1118; iso-2; iso-3. Fly (Austin). 2012;6(2):80-92.

59. Alva V, Nam SZ, Söding J, Lupas AN. The MPI bioinformatics Toolkit as an integrative platform for advanced protein sequence and structure analysis. Nucleic Acids Res. 2016;44(W1):W410-W415.

60. Liu CH, Chien CL. Molecular cloning and characterization of chicken neuronal intermediate filament protein $\alpha$-internexin. J Comp Neurol. 2013;521(9):2147-2164

61. Lahlali T, et al. Netrin-1 protects hepatocytes against cell death through sustained translation during the unfolded protein response. Cell Mol Gastroenterol Hepatol. 2016;2(3):281-301.e9.

62. Jin LQ, Zhang G, Jamison C, Takano H, Haydon $\mathrm{PG}$, Selzer ME. Axon regeneration in the absence of growth cones: acceleration by cyclic AMP. JComp Neurol. 2009;515(3):295-312.

63. Nasonkin IO, Lazo K, Hambright D, Brooks M, Fariss R, Swaroop A. Distinct nuclear localization patterns of DNA methyltransferases in developing and mature mammalian retina. J Comp Neurol. 2011;519(10):1914-1930.

64. Behrens TE, Berg HJ, Jbabdi S, Rushworth MF, Woolrich MW. Probabilistic diffusion tractography with multiple fibre orientations: What can we gain? Neuroimage. 2007;34(1):144-155.

65. Kezele I, Descoteaux M, Poupon C, Poupon F, Mangin JF. Spherical wavelet transform for ODF sharpening. Med Image Anal. 2010;14(3):332-342.

66. Tournier JD, Calamante F, Connelly A. Robust determination of the fibre orientation distribution in diffusion MRI: non-negativity constrained super-resolved spherical deconvolution. Neuroimage. 2007;35(4):1459-1472.

67. Mangin JF, Poupon C, Clark C, Le Bihan D, Bloch I. Distortion correction and robust tensor estimation for MR diffusion imaging. Med Image Anal. 
2002;6(3):191-198.

68. Tournier JD, Calamante F, Gadian DG, Connelly A. Direct estimation of the fiber orientation density function from diffusion-weighted MRI data using spherical deconvolution. Neuroimage. 2004;23(3):1176-1185.

69. Tuch DS, Reese TG, Wiegell MR, Makris N, Belliveau JW, Wedeen VJ. High angular resolution diffusion imaging reveals intravoxel white matter fiber heterogeneity. Magn Reson Med. 2002;48(4):577-582.

70. Jansons KM, Alexander DC. Persistent Angular Structure: new insights from diffusion MRI data. Dummy version. Inf Process Med Imaging. 2003;18:672-683.

71. Vo A, et al. Thalamocortical connectivity correlates with phenotypic variability in dystonia. Cereb Cortex. 2015;25(9):3086-3094.
72. Colby JB, Soderberg L, Lebel C, Dinov ID, Thompson PM, Sowell ER. Along-tract statistics allow for enhanced tractography analysis. Neuroimage. 2012;59(4):3227-3242.

73. Crawford JR, Howell DC. Regression equations in clinical neuropsychology: an evaluation of statistical methods for comparing predicted and obtained scores. JClin Exp Neuropsychol. 1998;20(5):755-762. 